\title{
Simulating high resolution inversion: decomposing the resolution function
}

Gerrit Toxopeus*, Steen Petersen†, Jan Thorbecke* and Kees Wapenaar*, * Delft University of Technology, Department of Geoscience. † Norsk Hydro

\section{Summary}

In order to correctly compare an impedance section obtained from industry practice inversion algorithms to the impedance of a synthetic geological model (velocity times density), the synthetic impedance values have to be filtered by an Angle filter. This is concluded after decomposing the resolution function into an Angle and Band limiting filter. The low computational costs of the method make it possible that an interpreter can iteratively use the method to minimize the mismatch between a real and a synthetic impedance section.

\section{Introduction}

The seismic experiment is an important tool for "understanding" the subsurface geology. A prerequisite for such an understanding is a clear relation between the seismic image and the complex Geological Depth Model. In the synthesis stage the geologist is concerned with the question how and to what extent geological details are visible in the seismic image. Previously we therefore invested the following relation

Depth Image $(\mathbf{x})=$ Image operator $\{$ Forward operator $\{$ Geological Depth Model (x) $\}$ \}.

To efficiently simulate migrated seismics, the combined operator was introduced by Schuster and $\mathrm{Hu},(2000)$ and extended for more complex geology by Toxopeus et. al., (2003).

$$
\begin{gathered}
\text { Depth Image }(\mathbf{x})=\text { Resolution function }\{\text { Geological } \\
\text { Depth Model }(\mathbf{x})\}
\end{gathered}
$$

where the Resolution function is the combination of the Image and Forward operator. The resolution function acts as a band limitation and angle filter to simulate prestack migrated seismics from a Geological Depth Model. So the relation can also be written as,

$$
\begin{gathered}
\text { Depth Image }(\mathbf{x})=\text { Band limitation filter }\{\text { Angle filter } \\
\{\text { Geological Depth Model }(\mathbf{x})\}\} .
\end{gathered}
$$

High resolution inversion aims at removing these filters from pre-stack migrated data. However, the industry practice is that most algorithms are working on a trace by trace basis (Oldenburg et al., 1988; van Riel and Berkhout, 1985), meaning that only the Band limitation filter is (partly) removed. Consequently, the derived impedance values from pre-stack depth migrated seismic cannot straight forwardly be compared to impedance values (density times velocity) directly computed from the synthetic Geological Depth Model. Two approaches can be followed to solve this. The first approach is to directly removing the resolution function from the migrated data as $\mathrm{Hu}$ and Schuster (2000) propose. The second approach is discussed in this paper, where the Angle filter is incorporated in simulating impedance values.

The paper proposes to extend the concept of the combined Forward and Image operator, such that the resolution function is decomposed into an Angle limitation and Band filter. Now by choosing different combinations of the Band limitation filter, either a pre-stack migrated image or a high resolution inversion result is simulated. Note that the illustrations in this paper are with $2-\mathrm{D}$ zero-offset data, but nothing prevents us from extension to (3-D) finite offset data.

\section{Decomposing the resolution function}

The idea of decomposing the resolution function into two new filters is based on analyzing the key-features of the previously introduced resolution function (Toxopeus et al., 2003) in the double Fourier domain. Step one is to look for the key features. In step two, the key-features are then used to directly construct two new filters. Figure 1 is meant to illustrate this process by showing in the upper part the combined operator and its two key features and in the lower part (below the black solid line) the construction of the two new filters using the derived key features.

Figure 1 (b) shows the zero-offset forward modeled response of a point scatterer buried at a depth of 2000 $\mathrm{m}$ in a homogeneous medium of $2000 \mathrm{~m} / \mathrm{s}$ and recorded with an aperture of $3000 \mathrm{~m}$ (Figure 1 (a)). In the forward modeling a wavelet with a frequency band between 5 to $70 \mathrm{~Hz}$ is used. Figure 1 (c) shows the migrated response, a resolution function. Using the discrete forward Fourier transform, both datasets are shown in the double Fourier domain (Figure 1 (d) and (e)). The $\omega$ axis of Figure 1 (d) is scaled with half the velocity to illustrate the principle of migration: mapping one frequency of Figure 1 (d) onto one circle of Figure 1 (e) (Stolt, 1978). Figure 1 (e) is now analyzed on its key characteristics. The first key-feature of this pineapple shaped filter is its limitation by the frequency content of the wavelet (denoted by the lines $\mathrm{f}_{\min }$ and $\left.\mathrm{f}_{\max }\right)$. Its second key-feature is, that it is constrained by the maximum angle of wave propagation (denoted by $\alpha_{\max }$ ). For a homogeneous medium this angle is directly related to the aperture, see for more details Schuster and $\mathrm{Hu}$ (2000). For a more complex geological model $\alpha_{\max }$ is obtained by ray tracing through the geological model, see Toxopeus et. al (2003) for more information. Note that in this example the pineapple shaped filter is symmetric, but depending on the position of the point scatterer, with respect to the aperture or a complex geological overburden, an asymmetric pineapple 


\section{Simulating high resolution inversion}

shaped filter can be obtained as well.

Now that the key-features are identified, the two new filters are directly constructed in the double Fourier domain. The first filter uses the maximum angle of wave propagation to form a stop/pass hourglass shaped filter (Figure 2 (f)). Note that this filter is only partly shown and (can) extend to Nyquist (see Figure 2 (b)). The second filter is circularly shaped and it contains the spectrum of the modeling wavelet along its radius (Figure $2(\mathrm{~g}))$. Multiplication (convolution in space) of the two new filters gives the pineapple shaped filter, which is also directly obtained from the combined operator (compare Figure 1 (e) and (h)). This shows that the resolution function (result of the combined operator) can be interpreted as acting as two separate filters. The filters are denoted as Angle and Band limitation filter, respectively. Summarized in other words: $\mathrm{A} \bullet \mathrm{B}=\mathrm{C}$. Finally, Figure 1 (i) and $(\mathrm{j})$ shows the inverse Fourier transform of the two filters.

\section{Simulating high resolution inversion}

High resolution inversion aims at removing the Angle and Band limiting filters from pre-stack migrated data. As stated in the introduction, the industry practice is that most algorithms are working on a trace by trace basis, meaning that only the Band limitation filter is (partly) removed. Consequently the high resolution inversion cannot straight forwardly be compared to the impedance values (density times velocity) directly computed from the Geological Depth Model. Now, the proper procedure of simulating a high resolution impedance section is illustrated using a case study of the Cook Formation (Offshore Norway). A representative high resolution inverted inline section is shown in Figure 3 (a). To better understand the small scale seismic features (see arrow in Figure 3 (a)) a detailed quantitative model of the sedimentary architecture is built with sedimentological data. Relevant impedance values derived from wire-line data were assigned to this facies model. For more information see Dalman et. al. (2004). Figure 3 (b) shows the impedance section, product of velocity and density. Compare the datasets to the inverted data and observe that the data is much too sharp. Next, the synthetic impedance section (velocity times density) is filtered according to the framework outlined in Figure 2 (a). As a first step only one Angle filter is created and applied to the whole Cook section. Note that this is an approximation, because the Angle filter should actually be laterally varying. After being applied to the impedance section in the double Fourier domain, Figure 3 (c) shows the results in the spatial domain. Note that wrap around effects are disturbing the sides of the dataset. The maximum angle of wave propagation is found by ray tracing through a synthetic complex overburden model with an aperture limitation of $3 \mathrm{~km}$. The Angle filter can also be cut by a frequency limitation. Figure 2 (c) shows an Angle filter with a cut off frequency $\left(\mathrm{f}_{\max }\right)$ of $70 \mathrm{~Hz}$. Applied to the Cook model, Figure 3 (d) shows the simulated band limited high resolution inversion results in the spatial domain. Note that the dataset is now more smeared, compared to Figure 3 (c), and seems to be more in accordance with Figure 3 (a), where a band limited high resolution inversion result is shown. Figure 3 (e) shows a simulated migrated section, where according to the actual processing parameters a wavelet with frequency band 5 to $70 \mathrm{~Hz}$ is used.

Like the combined operator concept, the computational costs of creating an Angle filter are also very low. Therefore, this will aid an interpreter in iteratively updating the Geological Depth model (given the geological constrains) and accordingly matching the simulated high inversion and pre-stack migration with the real inverted and pre-stack migrated results.

\section{Discussion}

The resolution function is decomposed into an Angle and Band limiting filter. By taking the right combination of the filters, either simulated migrated seismic or high resolution inversion results are simulated. These synthetic datasets can be properly compared to either high resolution inversion or a pre-stack depth migrated image. Due to the low computational costs an interpreter can iteratively use the method to minimize mismatches between real and synthetic sections.

\section{Acknowledgments}

The authors wish to thank Norsk-Hydro for financially supporting the research project. Acknowledged are Rory Dalman and Rick Donselaar for their help to build a synthetic model of the Cook Formation and Petar Angelov for helping with high resolution inversion of the real data.

\section{References}

Dalman, R., Donselaar, M., Dreyer, T., Luthi, S., and Toxopeus, G., 2004, Integrated sedimentological and forward seismic modelling of the Cook Formation (Oseberg Field, offshore Norway): AAPG.

Hu, J., and Schuster, G., 2000, Prestack migration deconvolution:, in 70th Ann. Internat. Mtg Soc. of Expl. Geophys., 984-987.

Oldenburg, D. W., Scheuer, T., and Levy, S., 1988, Recovery of the acoustic impedance from reflection seismograms:, in Inversion of geophysical data Soc. of Expl. Geophys., 245264.

Schuster, G. T., and Hu, J., 2000, Green's function for migration: Continuous recording geometry: Geophysics, 65, no. 01, 167-175.

Stolt, R., 1978, Migration by Fourier transform: Geophysics, 43, no. 1, 23-48.

Toxopeus, G., Wapenaar, C., and Petersen, S., 2003, Improved geological modeling and interpretation by simulated migrated seismics: Expanded Abstract of the 73rd Annual SEG Meeting.

van Riel, P., and Berkhout, A. J., 1985, Resolution in seismic trace inversion by parameter estimation:, in Geophysics Soc. of Expl. Geophys., 50, 1440-1455. 


\section{Simulating high resolution inversion}

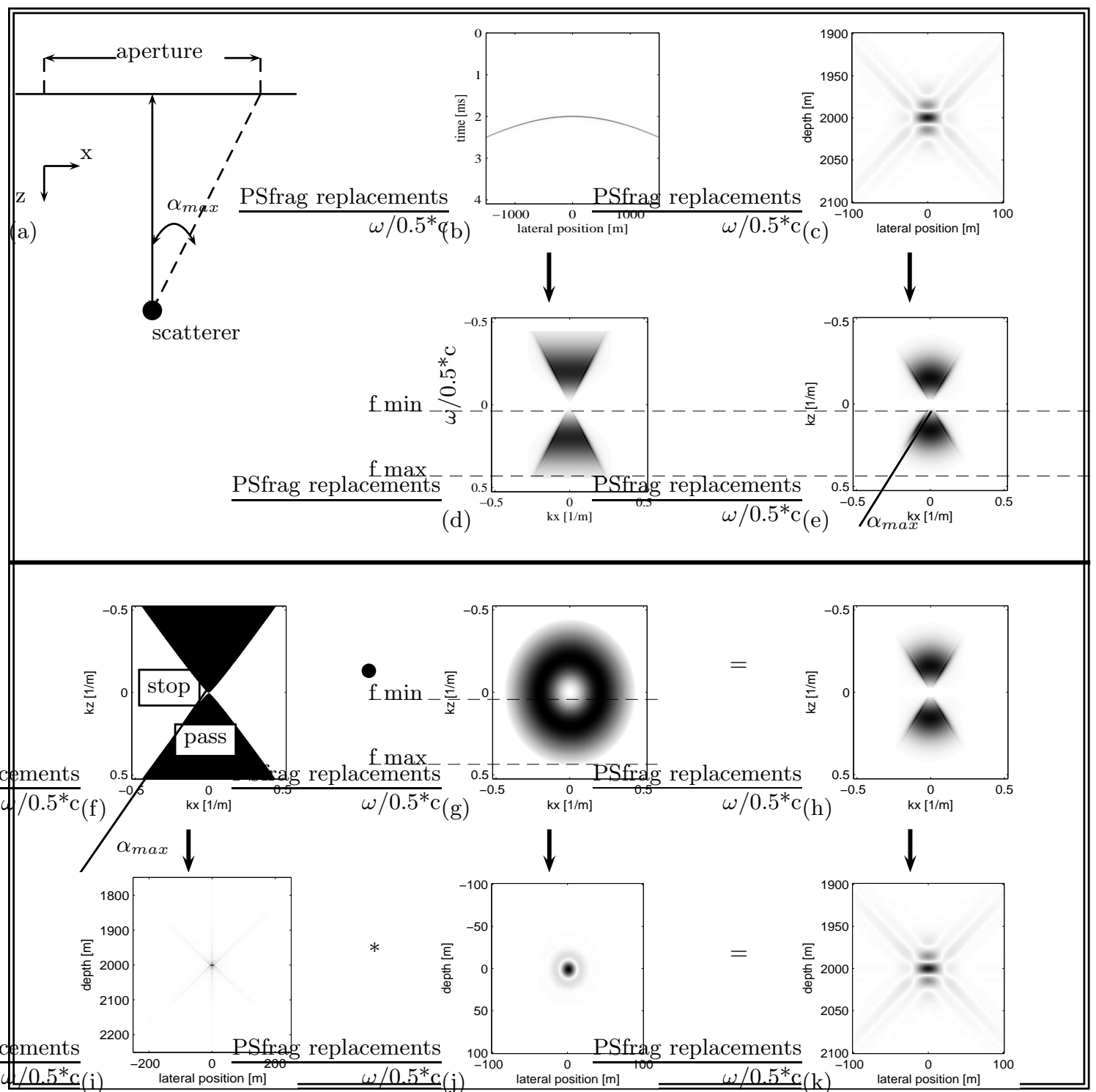

Fig. 1: Overview of the different filters. Above the black line the resolution function is modeled from the combined operator concept. Under the black line the resolution function is directly built in the double Fourier domain. Arrow, $\bullet$ and $*$ denote forward/inverse Fourier transform, multiplication and convolution, respectively. See text for more details.

3D Geological depth model

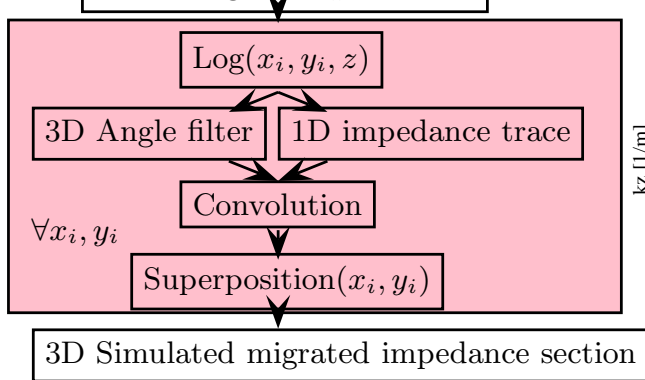

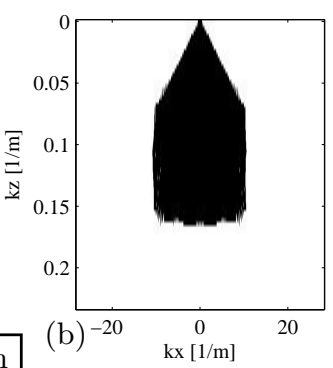

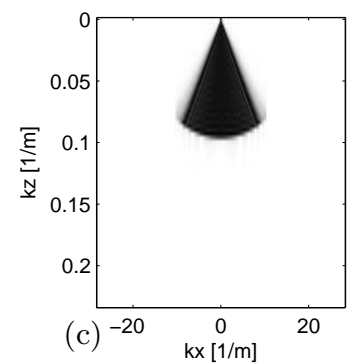

Fig. 2: (a) Framework to simulate high resolution impedance section. Summarized by convolving the Angle filter with an impedance trace, followed by superposition of all convolution products for all impedance traces. (b) Angle filter up to spatial Nyquist. (b) Angle filters with cut off frequency of $70 \mathrm{~Hz}$. 
Simulating high resolution inversion

lateral distance $[\mathrm{m}]$
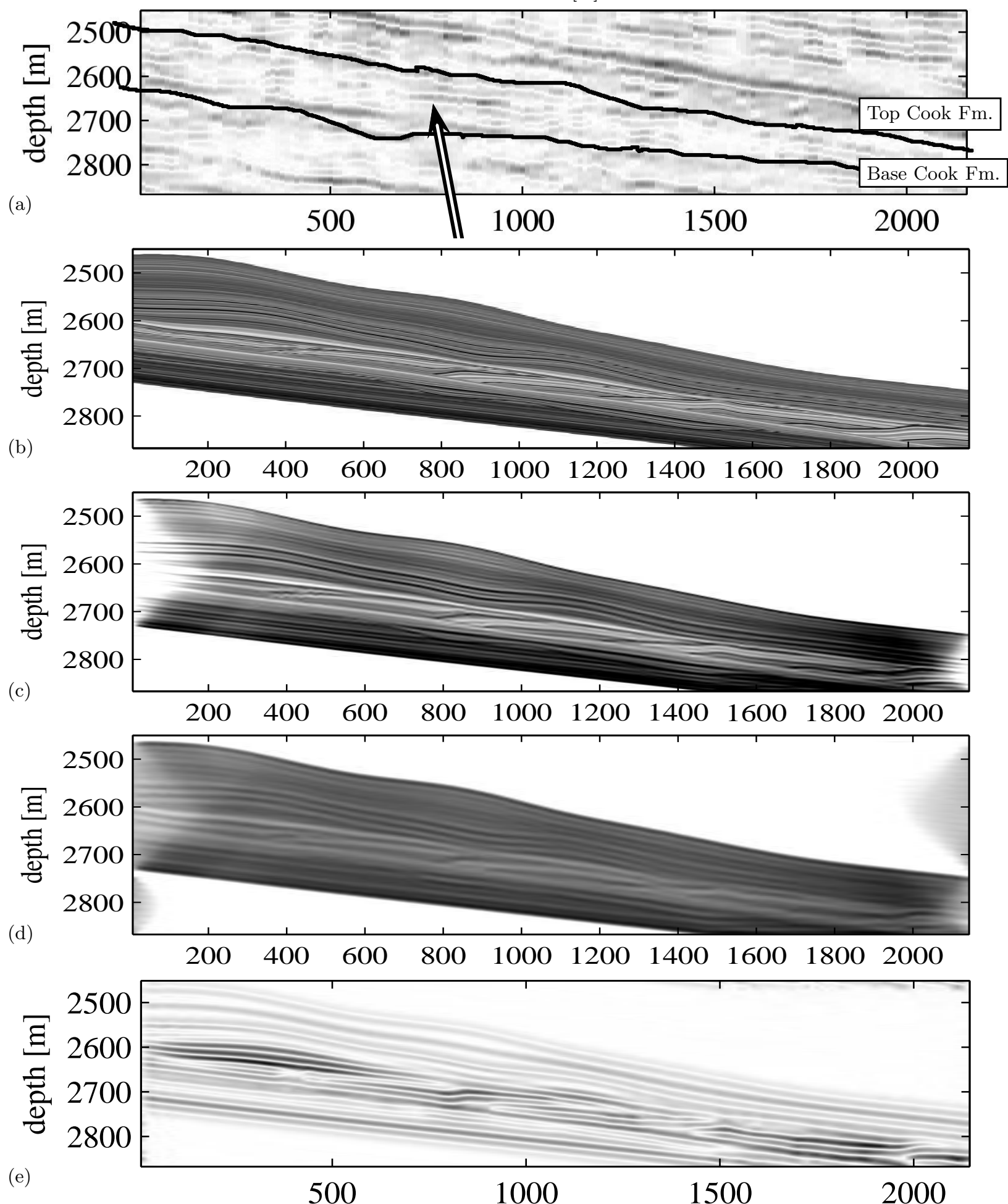

Fig. 3: (a) Impedance section of the Cook formation. Note the onlapping features (denoted by the arrow), which are of geological interest. (b) Synthetic impedance model (without Angle filter). (c) Synthetic impedance section, obtained with an angle filter up to Nyquist. (d) Synthetic impedance section, obtained with a band passed angle filter. (e) Simulated migrated section. 